\title{
Predominantly Increased Triglyceride-rich Lipoproteins Induced by Dietary Cholesterol Promoted Atherosclerosis Formation in LDL Receptor Knockout Golden Hamster
}

\section{Yuhui Wang ( $\nabla$ wangyuhui2009@bjmu.edu.cn )}

Institution of Cardiovascular Sciences, Health Science Centor, Peking University

Xiao Lin

Institution of Cardiovascular Sciences, Health Science Centor, Peking University

Ping Ma

Institution of Cardiovascular Sciences, Health Science Centor, Peking University

Chun Yang

Institution of Cardiovascular Sciences, Health Science Centor, Peking University

Jinjie Wang

Institution of Cardiovascular Sciences, Health Science Centor, Peking University

Kunxiang He

Institution of Cardiovascular Sciences, Health Science Centor, Peking University

Gonglie Chen

Institution of Cardiovascular Sciences, Health Science Centor, Peking University

Wei Huang

Institution of Cardiovascular Sciences, Health Science Centor, Peking University

Jianglin Fan

Department of Molecular Pathology, Graduate School of Medicine, University of Yamanashi

\section{Xunde Xian}

Institution of Cardiovascular Sciences, Health Science Centor, Peking University

George Liu

Institution of Cardiovascular Sciences, Health Science Centor, Peking University

\section{Article}

Keywords: Triglyceride-rich lipoproteins, Lipid metabolism, Atherosclerosis, LDL receptor, Golden hamster

Posted Date: June 25th, 2021

DOI: https://doi.org/10.21203/rs.3.rs-238928/v1 
License: (c) (i) This work is licensed under a Creative Commons Attribution 4.0 International License. Read Full License 


\section{Abstract}

Epidemiological investigation showed that triglyceride is an independent risk factor of cardiovascular diseases, but it is difficult to distinguish the effects of different lipoproteins by an experimental system in vivo, the role of triglyceride-rich lipoproteins (TRLs) in atherosclerosis have not been fully understood.

Here we found $\mathrm{LDL}$ receptor knock-out $\left(\mathrm{LDLR}^{-/-}\right)$hamsters have special characteristics of lipid profile for investigating effects of TRLs. Mixed hyperlipidemia in $\mathrm{LDLR}^{-/-}$hamsters after high cholesterol and high fat (HCHF) diet were marked by increasing of chylomicrons, VLDL and their remnants, but not LDL. Ezetimibe treatment significantly decreased these large particles containing $A p o B$ and ApoE without affecting LDL, leading to the dramatic reduction of plasma cholesterol and triglycerides. 40 days of HCHF diet feeding accelerated aortic atherosclerosis accompanied severe fatty liver, and ezetimibe treatment inhibited their development. Also, TRLs lowering inhibited the expression of vascular adhesion factors and lipid uptake of macrophage.

Our results suggest that golden hamster is a proper model for studying hypertriglyceridemia related diseases. In LDLR ${ }^{-/-}$hamster, TRLs showed independent atherogenicity by triggering inflammatory response of endothelial cells and the formation of foam cells from macrophages. And these TRLs clearance is mediated by LDL receptor. TRLs would be an important therapeutic target for atherosclerotic development with postprandial hyperlipidemia.

\section{Introduction}

Cardiovascular diseases (CVD) as a leading cause of death is associated with metabolic syndromes, including hyperlipidemia, non-alcohol fatty liver, diabetes, hypertension and obesity ${ }^{[1,2]}$. Atherosclerosis is the basic pathological process of CVD and hyperlipidemia is the most common risk or promoting factor.

According to the studies of apolipoprotein $E$ (ApoE) or low-density lipoprotein (LDL) receptor knockout mice so far, the formation of atherosclerotic plaques is mainly due to the infiltration and oxidation of LDL in the arterial wall ${ }^{[3]}$. Lots of studies have revealed the pivotal role of LDL-cholesterol (LDL-C) in mediating the progress of atherosclerosis. But the triglycerides are recognized having weaker effects on atherosclerosis, because that chylomicrons and VLDL may be difficult to enter vascular wall due to their large size $e^{[4]}$.

Syrian golden hamster has been one of the mostly used animals to study lipid metabolism because of its lipid profiling similar to human's ${ }^{[5-8]}$. Compared with mouse or rat, hamster expresses cholesteryl ester transfer protein (CETP), shows "LDL-based" lipoproteins profile in plasma, and responds sensitive to atherotropic or diabetic diets ${ }^{[6]}$. We have generated many genetically modified hamster models based on CRISPR/Cas9 technology, such as LDL receptor and lecithin cholesterol acyl transferase (LCAT) knockout hamsters $^{[9,10,11]}$, which phenotypes are similar to those of patients with the genetic mutations. 
Previously published studies have shown that blood lipids and glucose levels in hamsters were strongly regulated by diets, and these phenomena could not be found in mice or rats ${ }^{[12,13]}$. Interestingly, highly increasing levels of plasma triglycerides and TRLs fractions were found in these gene-knockout hamster models, especially with cholesterol rich diets ${ }^{[14]}$.

Although lowering LDL-c by statins is the primary treatment for patients with $\mathrm{FH}$ and hypercholesterolemia, it still has clinically valuable to reduce the absorption of exogenous cholesterol ${ }^{[15}$, 16]. Ezetimibe, which can inhibit NPC1L1 at the jejunal enterocyte brush border, has been proved to reduce area of atherosclerotic plaque in mice models ${ }^{[17-19]}$, or rabbits ${ }^{[20]}$. In many previous studies $[9,21,23]$, ezetimibe could completely reverse abnormal plasma lipids levels induced by HCHF diets in wild type and heterozygotes of $L D L$ receptor knockout $\left(\mathrm{LDLR}^{+/-}\right)$hamsters, suggesting a strong response of this species to dietary cholesterol absorption.

The extremely increased plasma lipids of $\mathrm{LDLR}^{-/-}$hamsters and its response to ezetimibe allowed us to explore the association between lipid metabolism and atherosclerosis in a different situation from the other hyperlipidemic animal models. In this study, we aimed to assess that TRLs, including chylomicrons, VLDL, and their remnants, facilitate the atherogenesis by animal model.

\section{Material And Methods}

\subsection{Animals}

Syrian golden hamsters and C57BL/6 mice were originally purchased from Beijing Vital River Laboratory Animal Technology Co., Ltd. (Beijing, China). LDL receptor knockout $\left(\operatorname{LDLR}^{-/}\right)$hamsters were generated by CRISPR/Cas9 genetic editing system in our lab and bred at SPF animal facility of Hebei ex\&invivo Biotechnology Co. as described previously ${ }^{[9]}$. $\mathrm{LDLR}^{-/-}$mice were provided by genetically modified animal model platform of Key Laboratory of Molecular Cardiovascular Sciences of National Science and Technology Ministry. All animals were fed with normal chow diet $20 \%$ protein and $4 \%$ fat; purchased from Beijing Ke'ao company, Beijing, China) or HCHF diet ( $0.5 \%$ cholesterol, $15 \%$ lard $(\mathrm{w} / \mathrm{w})$ based on chow diet) and water ad libitum. All animals were kept in a temperature-controlled environment on a lightdark cycle of 12L: 12D. The experimental procedures were handled according to the guidelines of the laboratory animal care (NIH publication no.85Y23, revised 1996) and approved by the Animal Care and Use Committee of the Peking university health science center (LA2015-012).

Female golden hamsters at the age of 12 months and female C57BL/ 6 mice at the age of 6 months were used in this study in order to induce atherosclerotic lesions more quickly. Thirty LDLR ${ }^{-/-}$hamster were divided into four groups: chow diet (CD), $n=5$; chow diet and ezetimibe (CD+EZE), n=5; HCHF diet (HFD), $n=10 ; \mathrm{HCHF}$ diet and ezetimibe (HFD+EZE), $n=10$. Twenty wild type (WT) hamster as the control were also divided into four groups as above identically $(n=5)$. The WT or LDLR ${ }^{-/-}$mice were divided into HFD and HFD+EZE groups $(n=10)$ as the controls of lipid lowering investigation for hamsters. Ezetimibe was administrated by gavage dissolved in saline and the saline as the control. The dose of ezetimibe for 
hamster was $2 \mathrm{mg} / \mathrm{kg} / \mathrm{day}$ and for mice was $3 \mathrm{mg} / \mathrm{kg} / \mathrm{day}$ which is hamster equivalent dose based on body surface area. Plasma was collected after 1 week and 2 weeks treatments for determination of plasma lipids levels and lipoprotein profiles. After 40 days, the hamsters were anesthetized by sodium pentobarbital and euthanized for tissues harvesting.

\subsection{Plasma lipids analysis}

Anticoagulated blood was collected after overnight fast after 2 weeks of HCHF diet and centrifuged (4000 $\mathrm{rpm}, 4^{\circ} \mathrm{C}, 10$ minutes) to separate plasma. The total cholesterol and triglycerides contents were measured using commercially available assay kits from Biosino Biotechnology and Science, Inc. (Beijing, China). Fractions of plasma lipoproteins was separated and collected using a ÄKTA fast protein liquid chromatography (FPLC) system (Amersham Biosciences). 100 $\mu \mathrm{L}$ pooled plasma from 5 aliquots of each group were followed by elution with buffer at a constant flow rate of $1 \mathrm{~mL} / \mathrm{min} .500 \mu \mathrm{L}$ per fraction were collected for triglycerides and cholesterol concentrations measurement. The plasma lipoproteins in $\mathrm{LDLR}^{-/-}$HFD group were too large to be processed with the column. This situation has not been encountered in other animal models during separation of plasma components. Because too many chylus in $\mathrm{LDLR}^{-/-}$hamster plasma after HCHF diet would damage the column (superpose $6 \mathrm{HR} 10 / 30, \mathrm{GE}$ ), the pooled plasma was centrifuged (15000 rpm, 30min) and the lower phase was used for loading to remove the very large particles, which accounted for approximately $50 \%$ of the estimated lipid concentrations in the pre-treatment samples. The triglycerides and cholesterol concentrations of total plasma and the lower phase were calculated to evaluate the quantity of large particles removed.

\subsection{Cell culture}

Human umbilical vein endothelial cells (HUVEC, 8000, ScienCell) were maintained in endothelial cell medium (1001, ScienCell) at $37^{\circ} \mathrm{C}$ under $5 \% \mathrm{CO} 2$ environment. The cells were subcloned into 6-well plates and equilibrated with $0.2 \%$ BSA-ECM for $6 \mathrm{~h}$. Then after added with $5 \%$ serum from different hamsters for 24 hours, the mediums were discarded and the cells were washed 3 times with PBS. The protein was extracted by RIPA Lysis Buffer (R0020, Solarbio) to prepare the samples for subsequent western blot analysis.

\subsection{Western blot analysis}

$1 \mu \mathrm{L}$ plasma or $20 \mu \mathrm{g}$ protein were prepared with the buffer containing sodium dodecyl sulfate (SDS) and dithiothreitol (DTT) by heating at $95^{\circ} \mathrm{C}$ for $10 \mathrm{~min}$. The samples were loaded to $10 \%$ or $6 \%$ SDS-PAGE gels and electrophoresed at $110 \mathrm{~V}$. Proteins were transferred into nitrocellulose membrane for $90 \mathrm{~min}$ at 220 $\mathrm{mA}$. Following blocking with $5 \% \mathrm{BSA}$, the membrane was hybridized with the following antibodies respectively: ApoB, ApoE, or ApoAl (ab20737, ab20874, ab20453, rabbit polyclonal IgG, Abcam). Plasma analysis was controlled by same volume of loading plasma and GAPDH (ab8245) as loading control for cell experiments. The target bands were visualized by incubation with horseradish peroxidase conjugated secondary antibodies followed by enhanced chemiluminescence detection (Molecular Imager Gel Doc XR System, Bio-Rad, Hercules, CA, USA). 


\subsection{Pathological analysis}

Tissues were fixed in $4 \%$ paraformaldehyde for $24 \mathrm{~h}$ and equilibrated in $20 \%$ sucrose for $24 \mathrm{~h}$. For oil red O (Sigma-Aldrich, St. Louis, MO, USA) staining, heart and liver were embedded in OCT, frozen at an approximate temperature of $-20^{\circ} \mathrm{C}$ and sectioned at $7 \mu \mathrm{m}$ with a freezing microtome (Leica) for preparation. The quantitative analysis of atherosclerosis was represented as the percentage of en face lesions area ratio to whole area of full length of aorta, and the total area of aortic root lesions by image $\mathrm{J}$ software. For H\&E, Sirius red and immunohistochemical staining, the tissue samples from aortic arch of all of hamsters after en face analysis were embedded in paraffin and sectioned at $3 \mu \mathrm{m}$ with a Leica microtome, and then following a standard protocol referring to our pathological platform.

Immunohistochemical staining was performed with VCAM-1 and a-SMA antibody (BA3840 and A03744, rabbit polyclonal IgG, Boster).

\subsection{Tissue lipid analysis}

Lipids extraction was referred to modified method of Bligh and Dyer ${ }^{[22]}$. Briefly, $100 \mathrm{mg}$ tissues were homogenized in $1 \mathrm{~mL}$ cold PBS. Then the lipids were extracted in $5 \mathrm{~mL}$ glass tubes to avoid polymer contamination by vortex with the same volume of chloroform/methanol (v:v=2:1) for 90 seconds and then centrifuged at $2000 \mathrm{rpm}$ for 20 minutes. The chloroform layer at lower phase was transferred using glass syringe and the rest was repeated the above operations. The collected chloroform layer was dried under nitrogen. Lipids dissolved with $3 \%$ TritonX-100 for analysis. The cholesterol and triglycerides contents were measured with the kits described above.

\subsection{Quantitative real-time PCR assay}

The total RNAs were extracted with Trizol reagent (Invitrogen, 12183555) and $50 \mu \mathrm{g}$ RNA was reversetranscribed by a commercial RT kit (Invitrogen, 18091200). Real-time PCR were performed using the AriaMx Real-Time PCR System with Top Green PCR Master Mix (TransGen Biotech, AQ131-01). The primers used in real-time PCR were listed in table1 and GAPDH as the internal reference.

\subsection{Quantification and Statistical Analysis}

All data were expressed as the mean $\pm \mathrm{SEM}$, and statistical tests are specified in figure legends. GraphPad Prism 7.0 software (GraphPad Software, La Jolla, CA, USA) was used for all statistical analyses. P-values of 0.05 or less were considered statistically significant.

\section{Result}

\subsection{Triglyceride-rich lipoproteins in hamster were regulated by both dietary cholesterol and LDL receptor significantly.}


As we have previously reported, hamster's plasma triglycerides level was sensitive to dietary cholesterol and easily elevated by HCHF diet ${ }^{[9-11,14,23]}$.

Here, we also found that, comparing with $\mathrm{LDLR}^{-/-}$mice, $\mathrm{LDLR}^{-/-}$hamsters had marked characteristics in significantly higher plasma total cholesterol and triglycerides levels in chow diet (Fig. 1A-B), and interestingly, HCHF diet dramatically increased plasma lipids levels, especially triglycerides levels. In addition, in wild type hamsters, HCHF diet also significantly increased the levels of plasma cholesterol and triglycerides (Suppl Fig. 1, A-B). Therefore, the golden hamster indeed is a special model for studying lipid metabolism and associated diseases being different from other rodents.

There were $30 \%$ decreasing of plasma total cholesterol levels in $\mathrm{LDLR}^{-/-}$hamsters after administration of ezetimibe with chow diet. Ezetimibe did not affect the plasma lipids levels in wild type hamsters and $\mathrm{LDLR}^{-/-}$mice when fed chow diet (Fig. 1A and Suppl Fig. 1A). While after HCHF diet, ezetimibe could completely inhibit the lipids elevation in both hamsters and mice. The big drop of the levels of plasma cholesterol by ezetimibe in hamster is impressive.

We also analyzed plasma lipoprotein profiles by FPLC to reveal changes of the different sizes lipoproteins. The results showed that in $\mathrm{LDLR}^{-/-}$hamsters HCHF diet mainly induced the increase of cholesterol in TRLs fraction. The effect of Ezetimibe was only to reduce cholesterol in TRLs fraction. (Fig. 1C). It was different in wild type hamsters that the changes happened in both TRLs and LDL particles (Fig. 1D). These changes of triglycerides in lipoproteins were consistent with the levels of cholesterol (the bottom panels of Fig. 1C, D).

These suggested that nutritional but not endogenous cholesterol might contribute to the increase of hamster's blood lipids, and the plasma TRLs fraction in $\mathrm{LDLR}^{-/-}$hamster, but LDL fraction in wild type hamster, are regulated by intestinal cholesterol. It's also evident that intestinal absorption cholesterol might mainly cleared by LDL receptor in hamster. The present and previous ${ }^{[8,23]}$ results all showed that, in rodents, golden hamster are more sensitive to dietary lipids and susceptible to hypertriglyceridemia. LDL receptor may play an important role in this process. LDL receptor is mainly involved in the clearance of triglycerides in plasma, and not in VLDL secretion (Suppl Fig. 2).

\subsection{Ezetimibe significantly blocked increase of ApoB48 and ApoE in plasma with HCHF diet.}

In order to further understand the variations of plasma lipoproteins profile in $\mathrm{LDLR}^{-/-}$and wild type hamsters, separated TRLs or $1 \mu \mathrm{L}$ plasma were analyzed by SDS-PAGE or western blot for apolipoproteins respectively. Consistent with lipids concentrations and lipoproteins fractions, both of $A p o B$ and $A p o E$ in the TRLs and plasma of $\mathrm{LDLR}^{-/-}$hamsters were significantly higher than those in wild type hamsters (Fig. 2A-B). In LDLR ${ }^{-/-}$hamster, HCHF diet caused a marked increasing of both ApoB48 and ApoE, and had no discernible effect on the level of ApoB100 and ApoAl. And ezetimibe mainly inhibited the increase of ApoB48 and ApoE (Fig. 2B). However, in wild type hamster, in addition to ApoB48 and ApoE, ApoB100 
and ApoAl also increased after HCHF diet, and ezetimibe could hinder them. As we known, ApoB100 and ApoB48 are derived from liver and intestinal synthesis respectively in hamster. The results further indicated that the increased cholesterol and TRLs in LDLR ${ }^{-/-}$hamster originated from intestinal source. These changes in hamster's lipoproteins are very like postprandial hyperlipidemia in humans. On other hand, it suggested that the clearance capacity of chylomicrons and their remnants might be poor in hamster, especially when LDL receptor is deficient. Consequently, LDL receptor should play an important role in metabolism of these lipoproteins.

\subsection{TRLs promoted atherosclerosis in the early stage on the basis of high LDL-C level}

Most of previous studies of atherosclerosis were based on mice and rabbits' models without severe hypertriglyceridemia ${ }^{[24-26]}$. In this study, LDLR $^{-/-}$hamsters have severe hypertriglyceridemia and their diet-influenced lipoproteins allowed us to investigate the atherogenic properties of TRLs particles. In above results, ezetimibe could affect the large particles of lipoproteins in LDLR ${ }^{-/-}$hamsters. Then, we compared the atherosclerotic formation with or without ezetimibe treatment to evaluate the role of TRLs. Referring to our previous study, the spontaneous atherosclerosis occurred in $\mathrm{LDLR}^{-/-}$hamsters more than 15 months old. Here, we induced 12 months old LDLR ${ }^{-/-}$hamster by 40 days' HCHF diet for faster formation of atherosclerotic lesions. The levels of total cholesterol and triglycerides exceeded 3000 $\mathrm{mg} / \mathrm{dL}$ in these $\mathrm{LDLR}^{-/-}$hamster with HCHF diet (Suppl Fig. 1C-D).

The pathological analysis of aortic root and full length of aorta en-face showed the early-stage atherosclerotic lesions in $\mathrm{LDLR}^{-/-}$hamsters (Fig. 3A, C), that were not found in wild type hamsters (Data not shown). We also found that the area of lesions decreased significantly by $75 \%$ in aorta root and $50 \%$ in full length after ezetimibe administration (Fig. 3B, D).

Further by sectioning of aortic arch for H\&E (Fig. 3E), we found that the plaques in aortic arch protruded into lumen and the most advanced plaque presented the instable character after $\mathrm{HCHF}$ diet, and ezetimibe inhibited these advanced plaques formation. However, comparing the same moderate lesions from LDLR ${ }^{-/-}$hamster with or without ezetimibe treatment, we found there was no significant difference in inflammatory reaction or smooth muscle migration by immunohistological staining (Suppl. Figure 3). In addition, when treating cultured HUVEC with the serum separated from LDLR ${ }^{-/-}$hamster with HCHF diet, we found that ICAM-1 and VCAM-1 expression significantly reduced when the cells were incubated with the serum after ezetimibe treatment (Fig. 3F, G).

To sum up the above data, we believe that high TRLs increased susceptibility to atherosclerosis in LDLR ${ }^{-/}$ - hamster with high LDL-c level. This may begin with the inflammatory response of endothelial cells to TRLs, and advanced with ox-LDL and remnants uptake by macrophages. 


\subsection{Severe accumulation of lipids in liver was significantly reduced by dietary cholesterol inhibition of ezetimibe}

After 40 days of HCHF diet and ezetimibe administration, we also found severe accumulation of lipids in LDLR-/- hamster and wild type hamsters' liver by H\&E and oil red $O$ staining, and lipids extraction. Ezetimibe could significantly reduce lipid deposition in the liver both in LDLR-/- hamster (Fig. 4A) and wild type hamster (Suppl Fig. 4). Consistent with pathological results, ezetimibe could reduce hepatic lipid deposition in $\mathrm{HCHF}$ diet feeding LDLR-/- hamster. Hepatic triglycerides reduced about $31 \%$ in HFD group treated with ezetimibe (Fig. 4B). Hepatic cholesterol reduced about $30 \%$ in CD group and $45 \%$ in HFD group respectively when treated with ezetimibe (Fig. 4C). That the lipids concentrations in liver not be reversed by ezetimibe like in plasma should be due to the upregulated synthesis (Fig. 5B) and downregulated VLDL secretion (Suppl Fig. 2B). Interestingly, H\&E, CD68, and TNF staining all showed that there were many inflammatory cells in the liver of LDLR ${ }^{-/-}$HFD group (Fig. 4A, D). Sirius red staining showed that HFD promoted fibrosis in the liver of LDLR ${ }^{-/-}$hamsters. Ezetimibe alleviated both the inflammatory responses and the fibrosis (Fig. 4D). In wild type hamsters, the same changes could be found in the liver (Suppl Fig. 4E).

We quantified the expression levels of several major genes involved in hepatic and intestinal cholesterol and FFA metabolism of LDLR-/- hamster (Fig. 5). Ezetimibe treatment upregulated the expression of HMGCoA synthetase and reductase significantly both in liver and intestine suggesting increase of endogenous cholesterol synthesis. The expression of ABCG5 was upregulated in liver and intestine by $\mathrm{HCHF}$ diet and ezetimibe downregulated the expression in liver but not in jejunum. SR-B1 was downregulated significantly by HCHF diet and recovered by ezetimibe in liver. SREBP1c and VLDL receptor were all upregulated by HCHF diet in LDLR-/- hamsters and recovered by ezetimibe in liver suggesting they responded to dietary cholesterol.

\section{Discussion}

The present study showed that the plasma lipids levels of a rodent, golden hamster, were much sensitively regulated by the dietary lipids ${ }^{[9,10,23]}$. Its mostly significant feature was that triglycerides levels can be remarkably increased by exogenous cholesterol in diet. The characteristics may make hamster be more useful to investigate lipid metabolic regulation and the associated diseases.

Our results showed that dietary lipids could predominantly increase large particles of plasma lipoproteins in hamster, especially in $\mathrm{LDLR}^{-/-}$hamster. Unlike in ApoE knockout mice, in these hamsters the elevation was particularly high. These large lipoproteins contributed to increasing levels of the plasma cholesterol and triglycerides (more than $80 \%$ ) in $\mathrm{LDLR}^{-/-}$hamster. Because of the ApoB editing only in intestine in hamster ${ }^{[7]}$, increasing ApoB48 and ApoE in $\mathrm{LDLR}^{-/-}$hamster after HCHF diet indicated the accumulation of chylomicron remnants in apolipoproteins analysis. Through SDS-PAGE in the TRLs of CD group, we also found that levels of ApoB100 and ApoE in $\mathrm{LDLR}^{-/-}$hamsters be significantly higher than those in 
wild type hamsters. Results in Ezetimibe treatment suggested that dietary cholesterol can lead to accumulation of TRLs and remnants in hamsters, which is improving by deficiency of LDL receptor. We thought that $\mathrm{LDLR}^{-/-}$hamster fed with HCHF diet could mimic postprandial hyperlipidemia. M van Heek, et $a^{[23]}$ had pointed out that ezetimibe alone may lead to a reduction in plasma cholesterol and triglycerides in humans with combined hyperlipidemia, such as obese insulin-resistant and/or type 2 diabetic patients. This is illustrated at least on a human-like animal model by our work.

Hamsters have plasma CETP activity which is a remarkable feature similar to human lipid metabolism. CETP activity could significantly increase in the postprandial state almost in parallel with the increase of plasma triglycerides $^{[32,33]}$. Subjects with CETP deficiency appeared to have lower levels of triglycerides and diminished remnant lipoprotein formation in the studies with rabbit model and human ${ }^{[34]}$. Therefore, the hamster was sensitive to HCHF diet for increasing TRLs and remnants as that in humans at least partially due to the CETP activity in plasma. Obviously, LDL receptor deficient dramatically further increased TRLs and their remnants associated with HCHF diet. To comparing LDLR ${ }^{-/-}$to wild type hamster, we found that LDL receptor knockout could delay the clearance of triglycerides and VLDL secretion (Suppl Fig. 2). And it suggested that LDL receptor can affect the clearance of TRLs. According to the analysis of apolipoproteins, mainly the clearance of remnants may be damaged. Hence, we supposed that LDL receptor should be also the receptor for chylomicron remnants.

In this study, we found the severity of atherosclerosis with 40-days HCHF diet represented the early-stage lesions. And these hamster models were characterized by mixed hyperlipidemia. The pathological results showed atherosclerotic lesions significantly decreased when ezetimibe inhibited the increasing of TRLs but not LDL. This is an evidence of an important role of dietary resource of TRLs in initial lesions. According to our previous study ${ }^{[14]}$, we considered that fat in diets have weak impacts on increasing hamster plasma lipids but could promote the absorption of cholesterol. The results of ezetimibe also suggested that dietary cholesterol might play an important role in triglycerides metabolism in hamster and it is an idea model for investigating pathophysiological mechanism of TRLs in human diseases.

Zilversmit proposed that chylomicrons or their remnants that increased in the postprandial plasma were major atherogenic lipoproteins ${ }^{[27]}$. In the present study, we offered the clear evidence to support the association between TRLs and the formation of atherosclerotic lesions in $\mathrm{LDLR}^{-/-}$hamsters with $\mathrm{HCHF}$ diet for their no elevation of LDL. The characteristics of LDLR-/- hamster can be used as an experimental system to show the role of TRLs in the development of diseases, but based on high LDL-C level in plasma. In wild type hamster, both of VLDL and LDL were significantly increased after HCHF diet, which both contributed to the development of atherosclerosis. Thus, the roles of VLDL and LDL were still indistinguishable in this model. Based on genetically modified mice models, such as $\mathrm{LPL}^{-/-}, \mathrm{GPIHBP}^{-/-}$, and $\mathrm{ApoC} 3$ transgenic mice, triglycerides were demonstrated to promote the atherosclerosis ${ }^{[28-30]}$. But $A p o B$ editing in both liver and intestine ${ }^{[31]}$ and insensitive to regulation of dietary lipids make these models could not to assess the role of different lipoproteins to mimic postprandial hyperlipidemia. 
TRLs and their remnants can lead to infiltration of monocytes by activating the inflammation and stress to impair endothelial function ${ }^{[35]}$. Relatively small size particles of TRLs remnants was also considered to be able to infiltrate into artery wall like LDL ${ }^{[36]}$. Subintimal lipoproteins may be modified and can be taken up via the receptors in macrophage, such as SR-A, LDL receptor, VLDL receptor, and so on, to promote the formation of foam cells and atherosclerotic lesions ${ }^{[37]}$. These studies help us to understand hypertriglyceridemia as an independent risk factor for cardiovascular diseases. We also found that the plasma with high TRLs and remnants could stimulate upregulation of ICAM- 1 and VCAM- 1 of cultured HUBEC (Fig. 3F, G), and then activated the activate mitogen-activated protein kinase (MAPK) signaling molecules ${ }^{[29]}$. It suggested that atherosclerosis may start with TRLs activating endothelial cells and being promoted by more monocyte's adhesion and lipoproteins infiltration. We supposed that TRLs also could be taken up by macrophages, and found that there were a lot of phagocytic lipids in hamster peritoneal macrophages after incubated with the plasma of HFD group (data not shown). The most severe plaques in $\mathrm{LDLR}^{-/-}$hamster with $\mathrm{HCHF}$ diet showed protuberant characteristics and morphological structure of lipid core. Therefore, because no other lipoprotein interference has been clearly excluded in other models, our study on hamster model confirmed the effects of TRLs on atherosclerosis, and the mechanisms involved are consistent with the common theories.

As we know, the liver plays an important role in lipids metabolism and fatty liver is usually associated with cardiovascular diseases. $\mathrm{LDLR}^{-/-}$hamster fed with HCHF diet also showed severe accumulation of lipids in liver. Interestingly, when ezetimibe inhibited absorption of cholesterol, lipid deposition in liver also was inhibited. It suggested that exogenous cholesterol played the important role not only in plasma lipid metabolism, but also in liver in hamster, and might be associated with atherosclerosis. The pathological sections also showed the focus of inflammatory cells in liver tissue, and it suggested that TRLs might induce inflammation in liver, consistent with the report of liver inflammation of LDLR ${ }^{-/-}$mice fed a cholesterol-rich diets ${ }^{[38]}$.

The expressions of hepatic genes involving in lipids metabolism were regulated by dietary cholesterol in $\mathrm{LDLR}^{-/-}$hamsters. Cholesterol synthesis genes in liver and intestine, such as Srebp1c, HMGCoA synthetase and reductase were down-regulated in LDLR-/-hamsters fed with HCHF diet and significantly up-regulated after ezetimibe treatment. It conforms to the ezetimibe's effects of regulation of cholesterol absorption and inhibition. ABCG5, a key gene for cholesterol efflux, was regulated by HCHF and ezetimibe also as expected. The upregulation of VLDLR after HCHF diet may be associated with elevated TRLs. But downregulation of SRB1 remain unclear in this situation. But anyway, we did not find that the LDLR-/mice showed these changes (Suppl. Figure 5) related to elevated triglycerides. The HCHF diet did not affect the expression of MTP suggested that elevated TRLs was not derived from VLDL secretion.

In conclusion, the changes of lipoproteins in $\mathrm{LDLR}^{-/-}$hamsters after $\mathrm{HCHF}$ diet and ezetimibe demonstrated that TRLs are starting and promoting factors in atherosclerosis. LDL receptor may also play an important role in triglycerides metabolism as the receptor of TRLs. With these characteristics of lipid metabolism, golden hamster is an ideal animal model for studying TRLs-related diseases. 


\section{Declarations}

\section{Credit authorship contribution statement}

X.L and P.M performed the most experiments. Y.W and X.L wrote the original draft. C.Y, J.W, K.H, and G.C participated in the experiments and provided some data. W.H, J.F, X.X gave methodological conducts including molecular biology and pathology. Y.W and G.L conceived the study and supervised experiments. G.L gave guidance in conceptualization and analysis.

\section{Funding source}

This work was financially supported by National Key Research and Development Program of China from Ministry of Science and Technology (2016YFE0126000) and National Natural Science Foundation (81770449 and 81570787) of China to Y.W. and Japan China Sasakawa Medical Fellowship to Y.W.

\section{Conflicts of Interest}

The authors indicated no potential conflicts of interest.

\section{Acknowledgments}

We thank Qiang Shen (Institute of Cardiovascular Science, Health Science Center, Peking University) for her technical assistance in morphology. We also thank Dr. Shouxiong Huang (University of Cincinnati) and Wei Zhang (People's Hospital of Peking University) for critical reading of the manuscript.

\section{References}

1. Clark, H. NCDs: a challenge to sustainable human development[J]. Lancet, 2013, 381(9866): 510511.

2. Roth, G.A., C. Johnson, A. Abajobir, et al. Global, Regional, and National Burden of Cardiovascular Diseases for 10 Causes, 1990 to 2015[J]. J Am Coll Cardiol, 2017, 70(1): 1-25.

3. Bartels, E.D., C. Christoffersen, M.W. Lindholm, et al. Altered metabolism of LDL in the arterial wall precedes atherosclerosis regression[J]. Circ Res, 2015, 117(11): 933-942.

4. Nordestgaard, B.G., and A. Tybjaerg-Hansen. IDL, VLDL, chylomicrons and atherosclerosis[J]. Eur J Epidemiol, 1992, 8 Suppl 1: 92-98.

5. Fernandez, M.L., T.A. Wilson, K. Conde, et al. Hamsters and guinea pigs differ in their plasma lipoprotein cholesterol distribution when fed diets varying in animal protein, soluble fiber, or 
cholesterol content[J]. J Nutr, 1999, 129(7): 1323-1332.

6. Castro-Perez, J., F. Briand, K. Gagen, et al. Anacetrapib promotes reverse cholesterol transport and bulk cholesterol excretion in Syrian golden hamsters[J]. J Lipid Res, 2011, 52(11): 1965-1973.

7. Liu, G.L., L.M. Fan, and R.N. Redinger. The association of hepatic apoprotein and lipid metabolism in hamsters and rats[J]. Comp Biochem Physiol A Comp Physiol, 1991, 99(1-2): 223-228.

8. Dietschy, J.M., S.D. Turley, and D.K. Spady. Role of liver in the maintenance of cholesterol and low density lipoprotein homeostasis in different animal species, including humans[J]. J Lipid Res, 1993, 34(10): 1637-1659.

9. Guo, X., M. Gao, Y. Wang, et al. LDL Receptor Gene-ablated Hamsters: A Rodent Model of Familial Hypercholesterolemia With Dominant Inheritance and Diet-induced Coronary Atherosclerosis[J]. EBioMedicine, 2018, 27: 214-224.

10. He, K., J. Wang, H. Shi, et al. An interspecies study of lipid profiles and atherosclerosis in familial hypercholesterolemia animal models with low-density lipoprotein receptor deficiency[J]. Am J Transl Res, 2019, 11(5): 3116-3127.

11. Dong, Z., H. Shi, M. Zhao, et al. Loss of LCAT activity in the golden Syrian hamster elicits proatherogenic dyslipidemia and enhanced atherosclerosis[J]. Metabolism, 2018, 83: 245-255.

12. Basciano, H., A.E. Miller, M. Naples, et al. Metabolic effects of dietary cholesterol in an animal model of insulin resistance and hepatic steatosis[J]. Am J Physiol Endocrinol Metab, 2009, 297(2): E462473.

13. Federico, L.M., M. Naples, D. Taylor, et al. Intestinal insulin resistance and aberrant production of apolipoprotein B48 lipoproteins in an animal model of insulin resistance and metabolic dyslipidemia: evidence for activation of protein tyrosine phosphatase-1B, extracellular signal-related kinase, and sterol regulatory element-binding protein-1c in the fructose-fed hamster intestine[J]. Diabetes, 2006, 55(5): 1316-1326.

14. Wang, J., K. He, C. Yang, et al. Dietary Cholesterol Is Highly Associated with Severity of Hyperlipidemia and Atherosclerotic Lesions in Heterozygous LDLR-Deficient Hamsters[J]. Int $\mathrm{J}$ Mol Sci, 2019, 20(14).

15. Toth, P.P., and M.H. Davidson. Cholesterol absorption blockade with ezetimibe[J]. Curr Drug Targets Cardiovasc Haematol Disord, 2005, 5(6): 455-462.

16. Kaye, T. Simvastatin with or without ezetimibe in familial hypercholesterolemia[J]. N Engl J Med, 2008, 359(5): 531-532; author reply 532-533.

17. Repa, J.J., S.D. Turley, G. Quan, et al. Delineation of molecular changes in intrahepatic cholesterol metabolism resulting from diminished cholesterol absorption[J]. J Lipid Res, 2005, 46(4): 779-789.

18. Umemoto, T., S. Subramanian, Y. Ding, et al. Inhibition of intestinal cholesterol absorption decreases atherosclerosis but not adipose tissue inflammation[J]. J Lipid Res., 2012, 53(11): 2380-2389. doi: 2310.1194/jlr.M029264. Epub 022012 Sep 029266.

19. Davis, H.R., Jr., D.S. Compton, L. Hoos, et al. Ezetimibe, a potent cholesterol absorption inhibitor, inhibits the development of atherosclerosis in ApoE knockout mice[J]. Arterioscler Thromb Vasc Biol, 
2001, 21(12): 2032-2038.

20. Gomez-Garre, D., P. Munoz-Pacheco, M.L. Gonzalez-Rubio, et al. Ezetimibe reduces plaque inflammation in a rabbit model of atherosclerosis and inhibits monocyte migration in addition to its lipid-lowering effect[J]. Br J Pharmacol, 2009, 156(8): 1218-1227.

21. Wu, Y., M.J. Xu, Z. Cao, et al. Heterozygous Ldlr-Deficient Hamster as a Model to Evaluate the Efficacy of PCSK9 Antibody in Hyperlipidemia and Atherosclerosis[J]. Int J Mol Sci, 2019, 20(23).

22. Byrdwell, W.C., H. Sato, A.K. Schwarz, et al. 31P NMR quantification and monophasic solvent purification of human and bovine lens phospholipids[J]. Lipids, 2002, 37(11): 1087-1092.

23. van Heek, M., C. Austin Tm Fau - Farley, J.A. Farley C Fau - Cook, et al. Ezetimibe, a potent cholesterol absorption inhibitor, normalizes combined dyslipidemia in obese hyperinsulinemic hamsters[J]. 2001(0012-1797 (Print)).

24. Lu, R., T. Yuan, Y. Wang, et al. Spontaneous severe hypercholesterolemia and atherosclerosis lesions in rabbits with deficiency of low-density lipoprotein receptor (LDLR) on exon 7[J]. EBioMedicine, 2018, 36: 29-38.

25. Niimi, M., D. Yang, S. Kitajima, et al. ApoE knockout rabbits: A novel model for the study of human hyperlipidemia[J]. Atherosclerosis, 2016, 245: 187-193.

26. Saulnier-Blache, J.S., R. Wilson, K. Klavins, et al. Ldlr(-)(/)(-) and ApoE(-)(/)(-) mice better mimic the human metabolite signature of increased carotid intima media thickness compared to other animal models of cardiovascular disease[J]. Atherosclerosis, 2018, 276: 140-147.

27. Zilversmit, D.B. Atherogenic nature of triglycerides, postprandial lipidemia, and triglyceride-rich remnant lipoproteins[J]. Clin Chem, 1995, 41(1): 153-158.

28. Zhang, X., R. Qi, X. Xian, et al. Spontaneous atherosclerosis in aged lipoprotein lipase-deficient mice with severe hypertriglyceridemia on a normal chow diet[J]. Circ Res, 2008, 102(2): 250-256.

29. Li, H., Y. Han, R. Qi, et al. Aggravated restenosis and atherogenesis in ApoCIII transgenic mice but lack of protection in ApoCIII knockouts: the effect of authentic triglyceride-rich lipoproteins with and without ApoCIII[J]. Cardiovasc Res, 2015, 107(4): 579-589.

30. Liu, X., J. Li, J. Liao, et al. Gpihbp1 deficiency accelerates atherosclerosis and plaque instability in diabetic Ldlr(-/-) mice[J]. Atherosclerosis, 2019, 282: 100-109.

31. Tadin-Strapps, M., L.B. Peterson, A.M. Cumiskey, et al. siRNA-induced liver ApoB knockdown lowers serum LDL-cholesterol in a mouse model with human-like serum lipids[J]. J Lipid Res, 2011, 52(6): 1084-1097.

32. Salerno, A.G., P.R. Patricio, J.A. Berti, et al. Cholesteryl ester transfer protein (CETP) increases postprandial triglyceridaemia and delays triacylglycerol plasma clearance in transgenic mice[J]. Biochem J, 2009, 419(3): 629-634.

33. Inazu, A., K. Nakajima, T. Nakano, et al. Decreased post-prandial triglyceride response and diminished remnant lipoprotein formation in cholesteryl ester transfer protein (CETP) deficiency[J]. Atherosclerosis, 2008, 196(2): 953-957. 
34. Mabuchi, H., A. Nohara, and A. Inazu. Cholesteryl ester transfer protein (CETP) deficiency and CETP inhibitors[J]. Mol Cells, 2014, 37(11): 777-784.

35. Yingchun, H., M. Yahong, W. Jiangping, et al. Increased inflammation, endoplasmic reticulum stress and oxidative stress in endothelial and macrophage cells exacerbate atherosclerosis in ApoCIII transgenic mice[J]. Lipids Health Dis, 2018, 17(1): 220.

36. Lusis, A.J. Atherosclerosis[J]. Nature, 2000, 407(6801): 233-241.

37. Tabas, I., K.J. Williams, and J. Boren. Subendothelial lipoprotein retention as the initiating process in atherosclerosis: update and therapeutic implications[J]. Circulation, 2007, 116(16): 1832-1844.

38. Wouters, K., P.J. van Gorp, V. Bieghs, et al. Dietary cholesterol, rather than liver steatosis, leads to hepatic inflammation in hyperlipidemic mouse models of nonalcoholic steatohepatitis[J]. Hepatology, 2008, 48(2): 474-486.

\section{Tables}

Table 1. The primers used in the real-time PCR.

\begin{tabular}{ccc}
\hline Gene & Forward & Reverse \\
\hline ABCG5 & CCATTCTGACTTACGGAGAGTTG & CAGGGGTAACCACAGTTATTGAA \\
ApoB & GTGTACGGCTTCAACCCTGA & TCAGGAATGGCCAGCTTGAG \\
CETP & TCCATAAGCTGCTCCTGCAC & GCCCTTGTGATGGGACTCAA \\
GAPDH & GACTCATGACCACAGTCCATGC & AGAGGCAGGGATGATGTTCTG \\
HMGCoA Synthetase & TCCTCCGTGGATTGCTCCTT & TCGGTCACTGTCTCCACCTT \\
HMGCoA Reductase & TGATGGGAGCTTGCTGTGAG & ACCAAGACCTATTGCCCTGC \\
& & \\
MTP & AGAGGAAAACCTGGACTCCTATG & AGCATTTTGGACATCAGATCACT \\
NPC1L1 & ATGGCCACTCACTGTCTTGG & CGTCGTGGAAAGCCTTCTCT \\
SRB1 & TGCCCGTCATCTACCAGTTG & TTTGGGACCCTACAGCTTGG \\
SREBP1C & GCGGACGCAGTCTGGG & ATGAGCTGGAGCATGTCTTCAAA \\
VLDLR & GCCTACCAGCACCACAGATT & GGTCACATTGATCCTTTGACACT \\
\hline
\end{tabular}

\section{Abbreviations}

ABCG5

ATP-binding cassette transporter G5

ApoA1

Apolipoprotein A1 
ApoB

Apolipoprotein B

ApoC3

Apolipoprotein C3

ApoE

Apolipoprotein $\mathrm{E}$

CETP

cholesteryl ester transfer protein

$\mathrm{CM}$

chylomicrons

CVD

cardiovascular diseases

FFA

Free fatty acid

GPIHBP1

glycosylphosphatidylinositol anchored high density lipoprotein binding protein 1

$\mathrm{HCHF}$

high cholesterol high fat

HDL

high density lipoprotein

HUVEC

human umbilical vein endothelial cells

LCAT

lecithin cholesterol acyl transferase

LDL

low density lipoprotein

LDLR

low density lipoprotein receptor

LPL

lipoprotein lipase

MTP

microsomal triglycerides transfer protein

NPC1L1

Niemann-Pick C1-Like 1

SR-A

scavenger receptor $A$

SRB1

scavenger receptor $\mathrm{B} 1$

SREBP1C

sterol regulatory element-binding protein $1 \mathrm{c}$ 
TRLs

triglyceride-rich lipoproteins

VCAM-1

Vascular cell adhesion molecule-1

VLDL

very low-density cholesterol

\section{Figures}
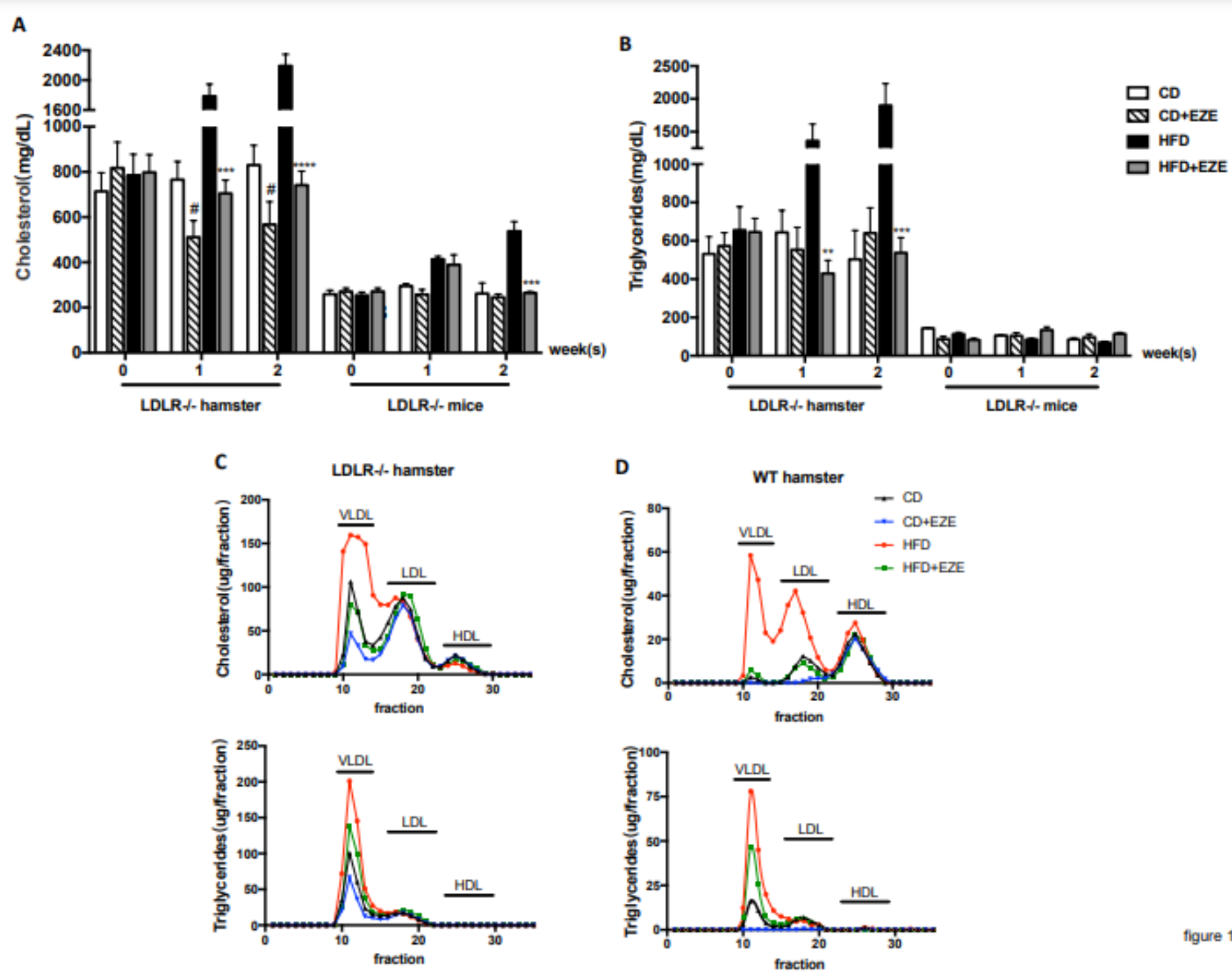

\section{Figure 1}

The responses to HCHF diet (HFD) and ezetimibe (EZE) in hamsters and mice. Total cholesterol (A) and triglycerides (B) level were determined by the plasma collected at 2 weeks from LDLR-/- hamster $(n=10)$ and mice $(n=5)$. Data are shown as means \pm SEM. \#, \#\#, \#\#\# CD+EZE vs CD or * **, *** HFD+EZE vs HFD represent $p<0.05,0.01$, and 0.001 respectively, two-way ANOVA with Dunnett's multiple comparisons test was performed. Lipids profiles of LDLR-/- (C) and wild type (D) hamster with different treatment for 2 
weeks were analyzed by FPLC. Each sample was pooled plasma of 5 hamsters and $100 \mu \mathrm{L}$ were loaded into the column. The cholesterol (upper) and triglycerides (bottom) of every collected fraction were determined and connected each point with a curve to make graph. Each peak represents CM/VLDL, LDL, $H D L$ fractions respectively from left to right.

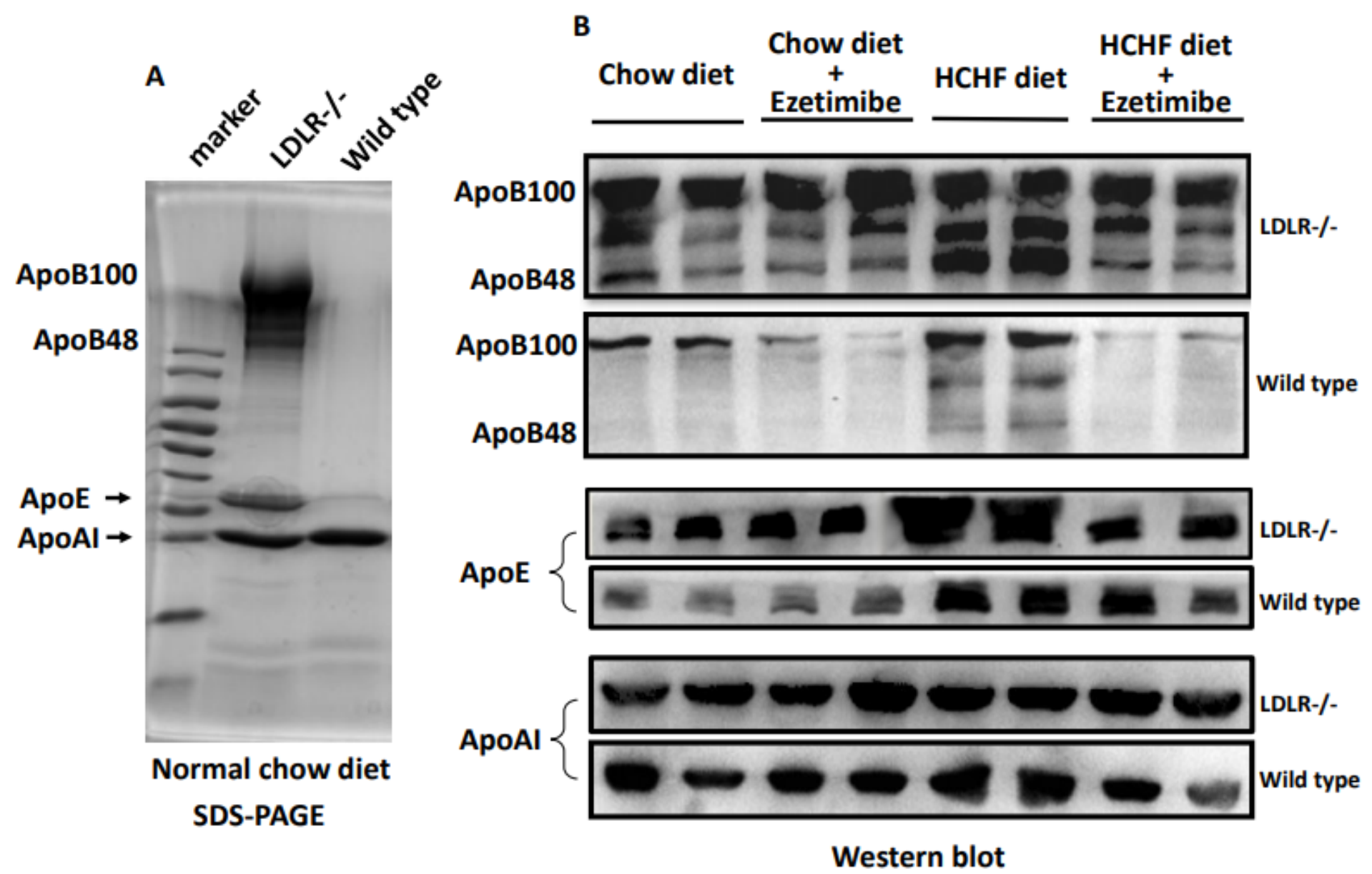

Figure 2

Apolipoproteins were analyzed by SDS-PAGE (A) and western blotting (B) for ApoB, ApoE and ApoAl in plasma of LDLR-/- and wild type hamster with or without ezetimibe administration for 2 weeks. CD means chow diet and HFD means HCHF diet. Separated TRLs from the fast plasma of hamsters with chow diet were used for SDS-PAGE analysis. $1 \mu \mathrm{L}$ plasma of each sample was used for analysis. 

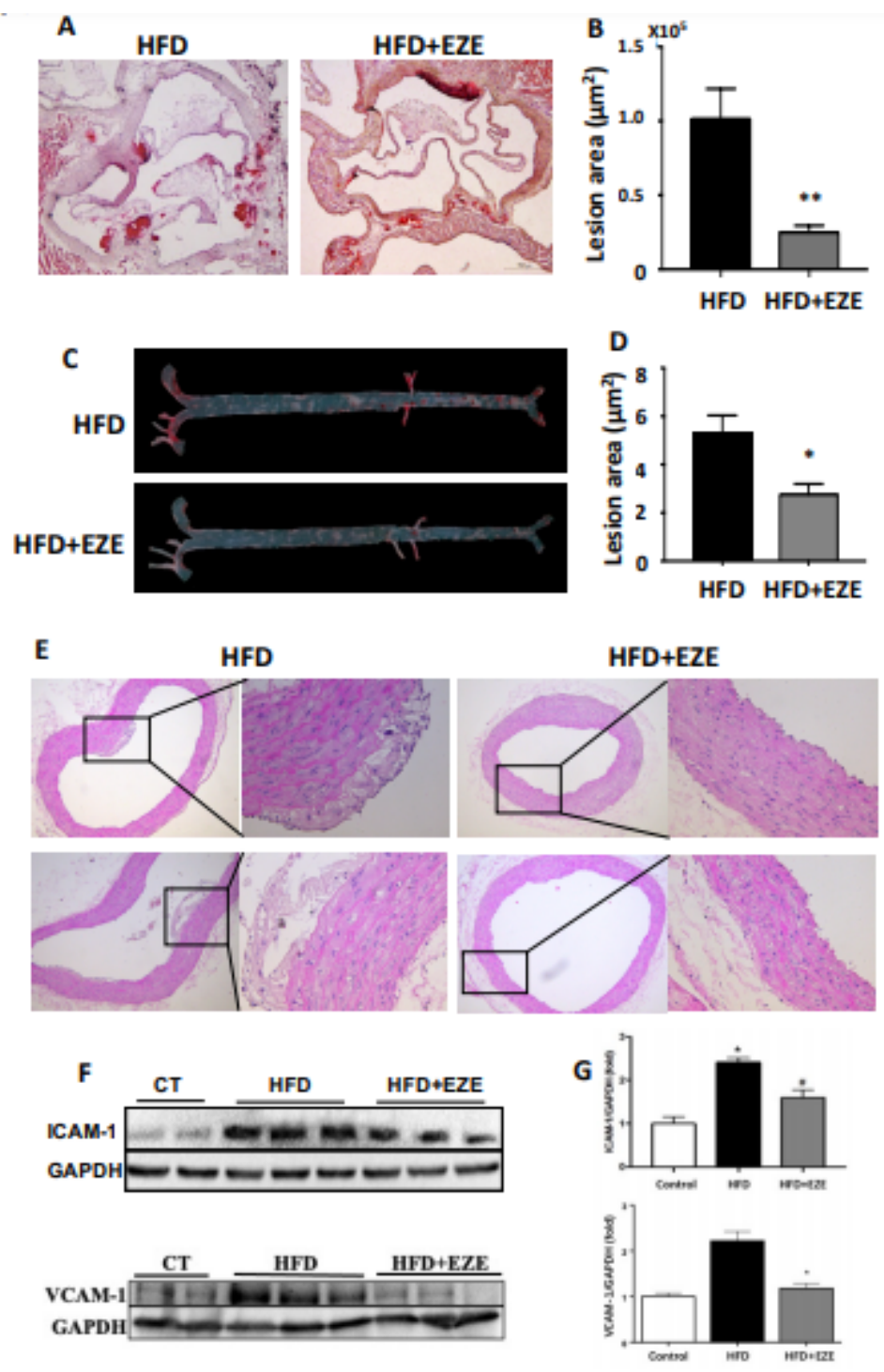

\section{Figure 3}

Ameliorative atherosclerosis in LDLR-/- hamster with ezetimibe treated in condition of HCHF diet feeding for 40 days. Representative pictures of the sections of aortic root (A) and en face analysis of whole length of aorta (C) by oil red $O$ staining $(n=10)$. Scale bars, $500 \mu m$. Total absolute oil red $O$ positive area in all sections of aortic root (B) and relative oil red 0-positive area of aortal en face $(n=10)$. Data are shown as means \pm SEM. ${ }^{*} p<0.05,{ }^{* *} p<0.01$ compared with HFD group, one-way ANOVA with Dunnett's multiple comparisons test was performed. E: Representative images of H\&E staining of the sections of aortic arch with 10x and 40x objective lens respectively. F: The expression of ICAM (upper) and VCAM-1 (lower) in cultured HUVECs analyzed by western blotting. The serum from normal hamsters as the control of normal expression, the expression of ICAM- 1 and VCAM- 1 incubated with the serum from HCFH diet hamsters (HFD) was upregulated and downregulated with the serum from HCHF diet hamsters with ezetimibe administration, and panel $\mathrm{G}$ is the quantitative bar charts. Data are shown as means $\pm \mathrm{SEM}$. Two-tailed unpaired t-test, \# HFD+EZE vs HFD, \#p<0.05, one-way ANOVA with Dunnett's multiple comparisons test was performed. 
A
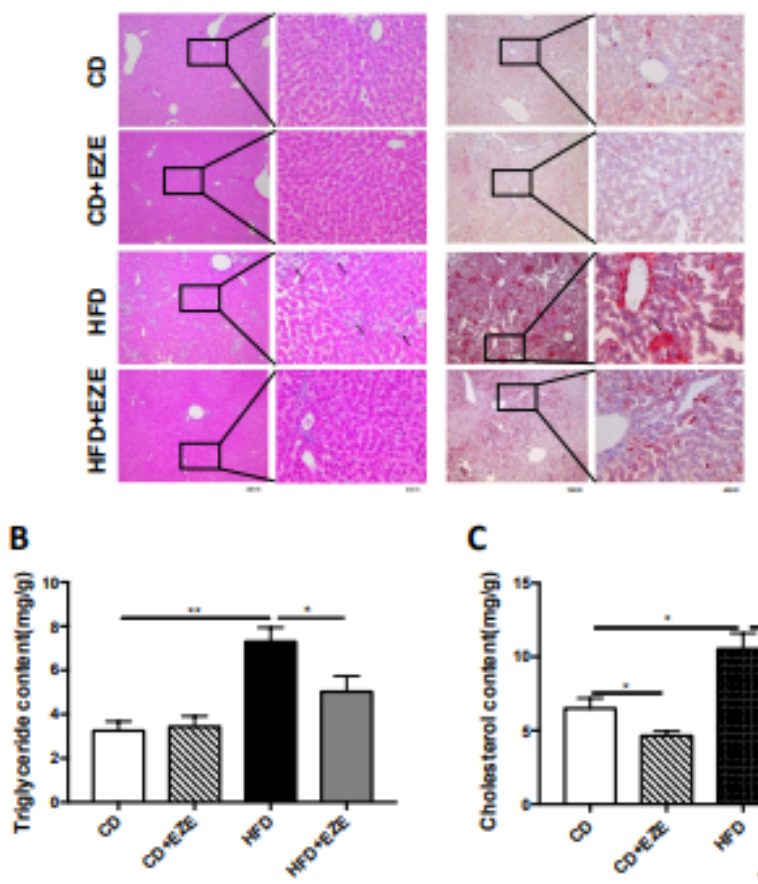

C
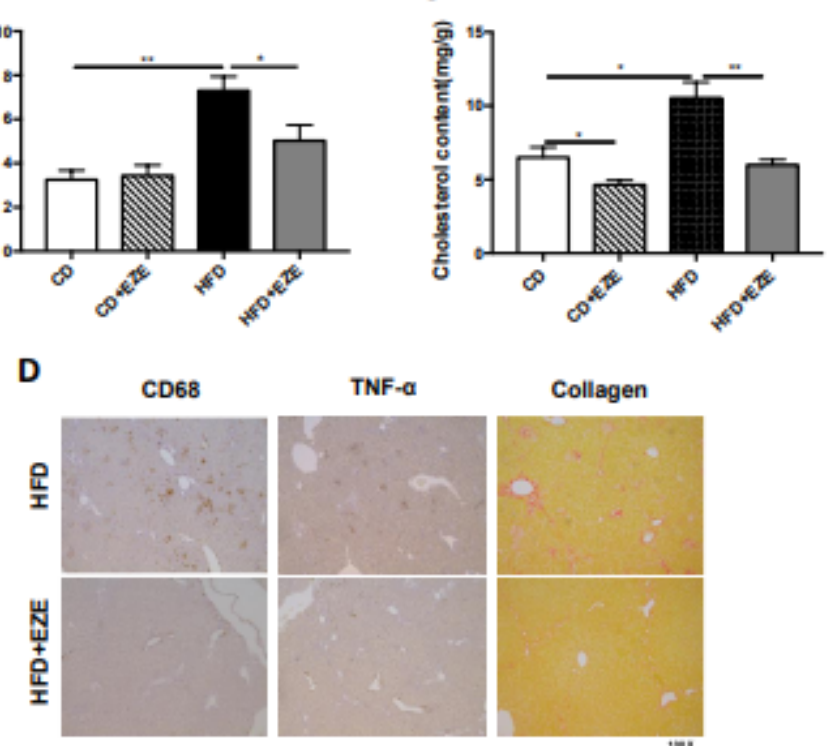

Collagen

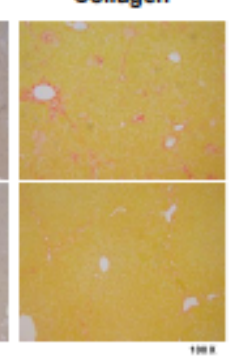

\section{Figure 4}

Hepatic morphological analysis and lipids extraction. LDLR-/- hamsters were fed chow diet (CD) or HCHF diet (HFD) for 40 days, meanwhile, administrated with ezetimibe (+EZE) or solvent. A: Representative pictures of liver cross-sections with H\&E and oil red 0 staining by $10 x$ and $40 x$ objective lens respectively. B: Hepatic triglycerides content $(n=5)$. C: Hepatic cholesterol content $(n=5)$. D: Representative immumohistochemical staining pictures of CD68 and TNF-a, and sirius-red staining pictures by 10x objective lens. Data are shown as means \pm SEM. Two-tailed unpaired t-test, ${ }^{*} p<0.05,{ }^{*} p<0.01$. 
A

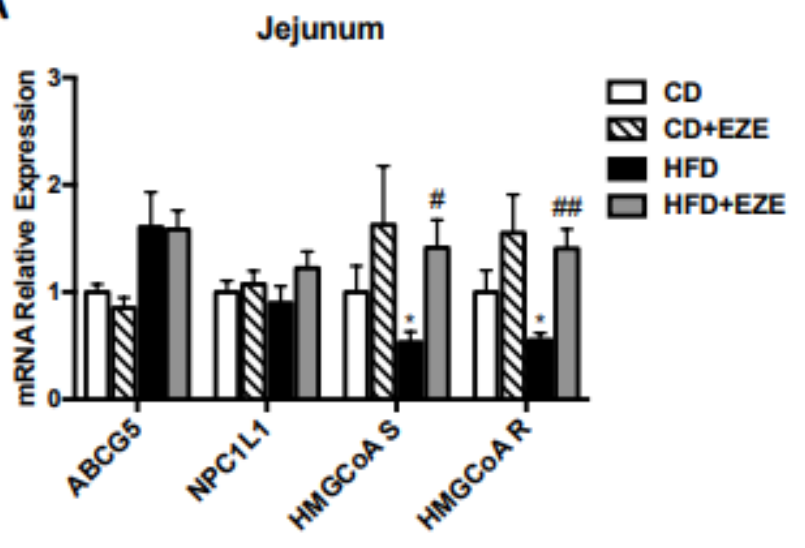

B

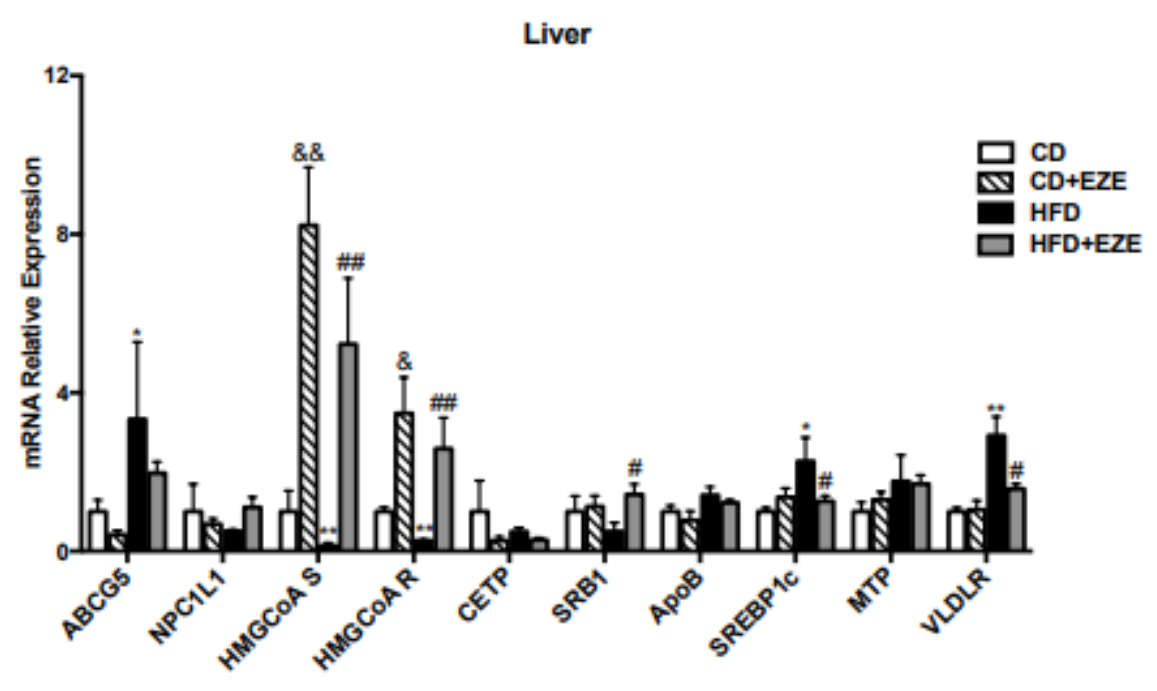

Figure 5

The expressions of the genes involved in lipid metabolism were determined by real-time PCR. LDLR-/hamsters were fed with chow diet (CD) or HCHF diet (HFD) for 40 days with ezetimibe (+EZE) or solvent treatment. Total mRNA was extracted from jejunum (A) or liver (B) for RT-PCR. Data are shown as times to $C D$ by means $\pm S E M, n=5$. Two-tailed unpaired t-test, * HFD vs $C D \otimes * p<0.05,{ }^{*} p<0.001 ; \& C D+E Z E$ vs $C D, \& p<0.05, \& \& p<0.001 ; \#$ HFD+EZE vs HFD, \#p<0.05, \#\#p<0.01.

\section{Supplementary Files}

This is a list of supplementary files associated with this preprint. Click to download.

- Supplementaryresults.docx 